\title{
Modeling and Verification of 802.16 MAC Protocol using Higher-Order Petri Nets
}

\author{
Ali Mohammed Meligy, Hani Mohammed Ibrahim, Amal Mohammed Aqlan \\ Menoufia University, Dept. of Mathematics, Faculty of Science, Egypt \\ meligyali@hotmail.com, hanimir78@yahoo.com, hamada_aqlan2010@yahoo.com
}

\begin{abstract}
In this paper, we present a new Petri Net (PN) approach to model the IEEE 802.16 MAC Protocol called Higher-Order Stochastic Reward Net (HOSRN) and through the application of the proposed rule. HOSRN is used as a modeling formalism as it readily captures the quality of service requirements of the traffic classes. We apply HOSRN to verify the effectiveness of QoS of the protocol using Reachability graph.
\end{abstract}

Index Terms - MAC; IEEE 802.16; QoS; Stochastic Reward Net(SRN); protocol verification; Higher-Order Petri Nets (HOPNs).

\section{INTRODUCTION}

The IEEE 802.16 medium access control layer (MAC) [1] protocol is designed for point-to-multipoint broadband wireless access (BWA) applications. It addresses the need for very high bit rates, both uplink (to the Base station) and downlink (from the Base station). The medium access control layer is capable of supporting multiple physical layer specifications optimized for the frequency bands of the application. The 802.16 specification accommodates MAC management messages that allow the base station to query the subscriber station. The challenge for BWA [2] networks is to simultaneously provide quality of service (QoS) to applications with very different characteristics. Hence, a proper resource allocation scheme for packet transmission is imperatively needed.

Increasing complexity of networks and the way in which they are used, has made it difficult to construct models that are analytically tractable. Stochastic Reward Nets (SRNs) are very useful in analytical modeling of complex networks. SRN refers to the augmented Stochastic Petri Net (SPN) with the reward description. System operations can be precisely described by means of a graph which translates into a Markova model. Properties such as Liveness and deadlock freeness make SRN a reliable analytical modeling tool. The authors in [2] presented a SRN approach to model IEEE 802.16 MAC with multiple traffic classes.

For communication protocol verification, a proper method must be selected so that properties and flows of protocol can be verified. Formal method can be used to ensure the correctness of models in the early software life cycle. There are two classes of formal methods which are formal specification method and formal verification method.

Formal specification describes properties of a system in a formal notation based on mathematical theory [3], therefore, the consistency of a system can be assured mathematically. There are several well known formal specification languages such as Z, LOTOS, VDM, Larch and Petri-Net. A Formal verification method is classified into model checking and theorem proving. Model checking transforms a logical model into automata and checks whether it has finite states. Theorem proving describes terminological form about the properties of requirements and proves the consistency of a system through mathematical inference rules.

The heuristic introduction of higher-order synaptic weights in neural networks has enabled to extend the concept of an arc in PN to a general sense that there exist higher-order arcs in PN [4]. the new class of Petri nets is called, higher-order Petri nets (HOPN).

In [5] Reisig and Meligy modify the higher-order Petri Nets $(H O P N)$ so that we can use classical analysis techniques. New graphical and mathematical methods for representing higher-order Petri Nets are suggested. they present a method to transform a $H O P N$ into a classical PN. they also show how to express the firing rule in case of HOPN using a matrix of weights. they study the possibility of reducing the set of higher-order matrices into a single matrix. Petri net is a powerful tool for modeling and analyzing communication protocols. Petri nets and communication protocols share many common properties. The modified HOPNs are used to model some data link protocols. As a solution to the problem of graph explosion in the case of complicated protocols, they suggest some simple graphical representation approaches.

Mahmoud, et. al. [6] considers HOPNs with transitions that have in degree $\leqslant 1$. As a prototype for our design approach we use Holton's Processor. We follow the top-down design, in which we refine the toplevel specification until we reach to the implementable level. For analyzing the HOPN, a theorem on the relationship between the potential friability of the goal transition and T-invariant is proved. The circuit synthesis corresponding to the HOPN is discussed. We use the properties of the HOPNs to check the validity of our design. 
In this paper, we propose a new Petri net called the Higher-Order stochastic Reward Net (HOSRN) by adding some amendments to the SRN Model, depending on the definition of Higher-Order Petri net and a proposed conditions. First, we show the proposed model, and then we check the validity of Model through reachability graph. The reachability graph of PN is the graph of markings that are reachable from other markings.

The rest of the paper is organized as follows: Section II presents a brief overview of IEEE 802.16 MAC. Introduction to Stochastic Reward Net is presented in Section III. Section V discusses Modeling and Verification of protocol. Conclusions are drawn in Section VI.

\section{RELATED WORK}

The 802.16 medium access control (MAC) layer [1] supports many different physical layer specifications, both licensed and unlicensed. Through the 802.16 MAC, every base station dynamically distributes uplink and downlink bandwidth to subscriber stations using time-division multiple access (TDMA). This is the basic difference from the earlier version of 802.11 MAC. 802.11 MAC operating through the use of carrier sensing mechanisms does not provide effective bandwidth control over the radio link. The MAC comprises three sub layers these are service specific convergence sub layer, medium access control common part sub layer (MAC CPS), and privacy sub layer. The service specific convergence sub layer (CS) provides transformation or mapping of external network data, received through the $\mathrm{CS}$ service access point (SAP), into MAC SDUs received by the MAC common part sub layer (MAC CPS) through the MAC SAP. This includes classifying external network service data units (SDUs) and associating them to the proper MAC service flow and connection identifier (CID). The MAC CPS provides the core MAC functionality of system access, bandwidth allocation, connection establishment, and connection maintenance. The MAC also contains a separate privacy sub layer providing authentication, secure key exchange, and encryption. Data, physical layer (PHY) control, and statistics are transferred between the MAC CPS and the PHY via the PHY SAP. The PHY may include multiple specifications, each appropriate to a particular frequency range and application.

There are a limited number of research which using Petri net for modeling of IEEE 802.16 MCA protocol. in paper [2] presents stochastic reward net (SRN) approach to analyze the performance of IEEE 802.16 MAC with multiple traffic classes. The model includes priority, pre-emption and time-out characteristics of traffic classes. The model can be extended to include more than three traffic classes. The model can be generalized to incorporate multiple SSs.

The authors in [7] presented a SRN approach to model IEEE 802.11 DCF with on-off traffic model.
Performance metrics such as mean delay and average system throughput have been evaluated. IEEE 802.16 MAC Protocol.

IEEE 802.16 system consists of two kinds of fixed stations: subscriber station (SS) and base station (BS) [2]. All communication in the network is regulated by BS. Two direction of communication path exists between BS and SS: uplink (from SS to BS) and downlink (from BS to SS). IEEE 802.16 MAC defines QoS signaling mechanisms and functions that control BS and SS data transmissions. Two modes of sharing the wireless medium is possible: Point-to-Multipoint (PMP) and Mesh. In PMP, BS serves a set of SS in a broadcast manner. Coordination of transmissions from SSs is done by BS. In mesh mode, organization of nodes is in ad hoc manner and communication exists between SS. In this paper, we focus on PMP mode.

In order to meet the QoS requirements of multimedia applications, the IEEE 802.16 MAC define four different scheduling service flows (Unsolicited Grant Service (UGS), Real-time Polling Service (rtPS), Nonreal-time Polling Service (nrtPS) and Best Effort (BE)). The SS maintains separate connection for each service flow. The allocation of bandwidth by the BS to SS is based on two modes: grant per subscriber station (GPSS), (where, the SS obtains aggregate bandwidth for all its individual flow and in turn reallocates the bandwidth to each flow individually) and grant per connection (GPC), (where, the bandwidth allocation by $\mathrm{BS}$ is made on per flow).

The IEEE 802.16 advantages includes variable bit rate and high data rate, last mile wireless access, point to multipoint communication, large frequency range and QoS for various types of applications [8].

\section{INTRODUCTION TO STOCHASTIC REWARD NET (SRN)}

Stochastic reward net (SRN) is an extension of Petri net $(\mathrm{PN})$ which is a high level description language for formally specifying complex systems. SRN has the advantage of specifying a real-world model in a compact and intuitive way [9]. A PN is a bipartite directed graph with two types of nodes: places and transitions. Each place may contain an arbitrary (natural) number of tokens. For a graphical presentation, places are depicted as circles, transitions are represented by bars and tokens are represented by dots or integers in the places. Each transition may have zero or more input arcs, coming from its input places; and zero or more output arcs, going to its output places. A transition is enabled if all of its input places have at least as many tokens as required by the multiplicities of the corresponding input arcs. When enabled, a transition can fire and will remove from each input place and add to each output place the number of tokens corresponding to the multiplicities of the input/output arcs. A marking depicts the state of a PN which is characterized by the assignment of tokens in all the places. With respect to a given initial marking, the reach 
ability set is defined as the set of all markings reachable through any possible ring sequences of transitions, starting from the initial marking.

Generalized stochastic Petri nets (GSPNs) extend the PNs by assigning firing time to each transition. Transitions with exponentially distributed ring times are called timed transitions while the transitions with zero ring times are called immediate transitions. A marking in a GSPN is called vanishing if at least one immediate transition is enabled; otherwise it is called a tangible marking.

In order to make more compact models of complex systems, several extensions are made to GSPN, leading to the SRN. One of the most important features of SRN is its ability to allow extensive marking dependency. In an SRN, each tangible marking can be assigned with one or more reward rate(s). Parameters such as the ring rate of the timed transitions, the multiplicities of input/output arcs and the reward rate in a marking can be specified as functions of the number of tokens in any place in the SRN. Another important characteristic of $\mathrm{SRN}$ is the ability to express complex enabling/disabling conditions through guard functions. This can greatly simplify the graphical representations of complex systems. For an SRN, all the output measures are expressed in terms of the expected values of the reward rate functions.

\section{MODELING AND VERIFICATION OF IEEE 802.16 MAC PROTOCOL USING HIGHER-ORDER STOCHASTIC REWARD NET}

\section{A. Modeling Protocol using HOSRN}

A typical IEEE 802.16 network consists of multiple BSs. Each BS covers several SSs. Every SS is associated with multiple queues corresponding to different traffic classes. We model a single SS with three queues corresponding to rtPS, nrtPS and BE traffic classes as shown in Figure 1. The SS is assigned aggregate bandwidth by the BS. The three queues contend for bandwidth from the SS. The model incorporates priority, pre-emption and timeout characteristics of the queues. Tables I-III lists the various places, transitions and the meaning associated with each of them.

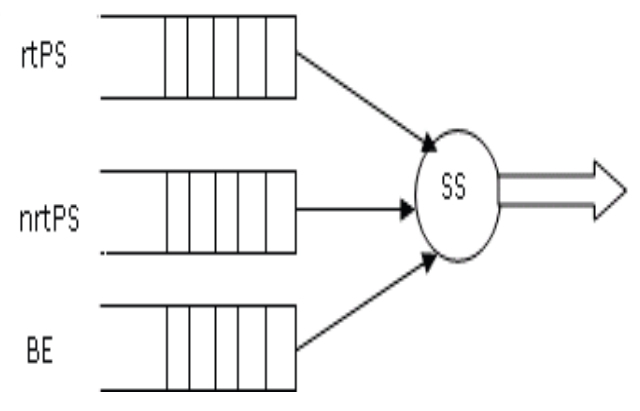

Figure 1: System model
TABLE I. LIST OF PLACES

\begin{tabular}{|c|c|}
\hline Place & Meaning \\
\hline Cap & Total available bandwidth \\
\hline$u s g_{i}$ & Number of channels currently in use \\
\hline$q_{i}$ & Packets in buffer \\
\hline
\end{tabular}

TABLE II. LIST OF TIMED TRANSITIONS

\begin{tabular}{|c|c|}
\hline $\begin{array}{c}\text { Timed } \\
\text { Transition }\end{array}$ & Meaning \\
\hline$u s r_{i}$ & Packet arrival at rate $\lambda_{i}$ \\
\hline$e n d_{i}$ & $\begin{array}{c}\text { Departure of packets after } \\
\text { service at rate } u_{i}\end{array}$ \\
\hline Time_o $o_{i^{*}}$ & $\begin{array}{c}\text { Removal of time out packets at } \\
\text { rate } u o_{-i}\end{array}$ \\
\hline
\end{tabular}

TABLE III. LIST OF IMMEDIATE TRANSITIONS.

\begin{tabular}{|c|c|}
\hline $\begin{array}{c}\text { Immediate } \\
\text { Transition }\end{array}$ & Meaning \\
\hline chch $k_{i}$ & $\begin{array}{c}\text { Priority transition checking } \\
\text { availability of channel }\end{array}$ \\
\hline pre_empt $_{i, j}$ & Enable pre-emption \\
\hline
\end{tabular}

Before we convert any model from classical Petri net and its variant types to HOPN, we must ensure the validity of all of the following conditions:

- First: If there exist at least two of transitions that have the same output place(s).

- Second: If there exist one of the previous transitions that have at least two of the incoming arcs.

- Third: If all previous transitions have the same type (timed or immediate transitions).

Then we apply the following steps:

1. For each transition contain at least two of incoming arcs. An arcs become a single arc, as in the follow figure:

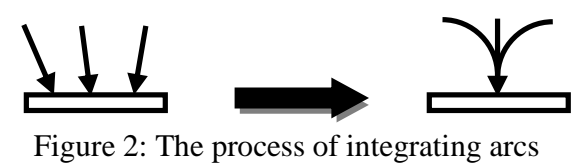

2. Merge all transitions to a single transition, as in Figure 3. 


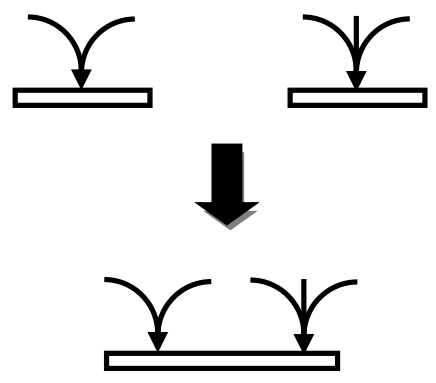

Figure 3: The process of integrating transitions

Now we have a new model of HOPN.

In [2] Geetha and Jayaparvathy present the SRN model for a SS with three queues in the IEEE 802.16 MAC protocol. We are now applying the rule previous on this model.

By applying the conditions, we find that:

- Transition $\left(\right.$ prompt $\left._{13}\right)$ and transition ( prompt $\left._{12}\right)$ have the same output places ( $c a p$ and $q_{1}$ ).

- Both transitions (prompt 13 and prompt $_{12}$ ) have two of the incoming arcs.

- Both transitions ( prompt $_{13}$ and prompt $_{12}$ ) have the same type (Immediate Transitions).

All conditions have been achieved and then apply the steps (1 and 2), we note that:

From Figure 4 produces a new model from HOPN with SRN called Higher-Order Stochastic Reward Net (HOSRN). Transition usri $(\mathrm{i}=1,2,3)$ generates packets at a given rate $\lambda \mathrm{i}$ and deposits them into place qi [2].

An inhibitor arc with cardinality bufi is needed to ensure that the number of packets waiting to enter the current queue is finite. If all channels are busy, the data packets are buffered in qi with buffer size bufi (in our study assume that bufi $=1$ ).

Transition chchki are modeled as priority transitions. Lower integer value indicates higher priority level. A priority transition is enabled only if no other higher priority transition is enabled. Since, chchk1 is assigned lowest value; class1 has highest priority to gain access to channel, followed by class 2 and class 3 . Firing chchki transfers a packet from qi to usgi indicating the packet is being served. After completion of service time, transition endi is fired and the channel is returned to the central pool. Note that chchki are modeled as immediate transitions since they represent activity that does not imply time dependency.

Transition prempt1, 23 are immediate transitions used to model pre-emption. Prempt1, 23 is enabled when packets are available in places $q 1$ and usgj $(j=2,3)$ at the same time. Arc connecting prempt1, 23 indicates removal of packet from usgj, and returning the channel to central pool of channels. Hence, firing prempt1, j preempts classj and enables class 1 to access the resource.

The channels available in the central pool of resource are shared by the traffic classes on arrival of data packets and returned to the pool on completion of service. At higher traffic loads, the available channels become insufficient to meet the bandwidth requirement. Under such conditions, packets in buffer wait for availability of resource [see ref.2].

\section{B. Model Verification}

We use Petri Net widely for the verification of network protocols. Petri Net describes a system using state transition approach and all possible states can be traced using reachability analysis [8]. However, state transition approach causes a problem, called state explosion as the number of states increases. The primary strength of Petri Net provides the abilities of exploring the states and representing states graphically. The places, the elements of Petri Net model, represent states of nodes in the network. The transitions symbolize messages of the protocols; moreover, the flows of tokens indicate variations with control messages or data among nodes related to network protocols.

Petri Net has the following properties for protocol verification: reachability, boundedness, liveness and reversibility.

Reachability means that a certain state can be reachable from the initial state. Boundedness is used to model limits on available system resources. The property of liveness is used to model that the system can never lock up a deadlock. Reversibility is used to represent re-initialization and cyclic behavior.

In this section, we describe the model verification for the IEEE 802.16 MAC protocol using HOSRN based on the Reachability graph. cap place is the initial place for the different nodes; the black points in cap place is the initial marking. each place transit into next place through a transition.

Figure 5 shows the reachability graph for the validation of compatibility such as checking protocol deadlock and liveness. We rewrite symbols transitions $(\mathrm{t} 1=\mathrm{usr} 1, \mathrm{t} 2=$ chchk $1, \mathrm{t} 3=$ time $\mathrm{o} 1, \mathrm{t} 4=\mathrm{usr} 2, \mathrm{t} 5=$ chchk $2, \mathrm{t} 6=$ time $\mathrm{o} 2, \mathrm{t} 7=\mathrm{usr} 3, \mathrm{t} 8=\operatorname{chchk} 3, \mathrm{t} 9=$ prempt $1,23, \mathrm{t} 10=$ end $1, \mathrm{t} 11=$ end $2, \mathrm{t} 12=$ end $3, \mathrm{t} 13=$ prempt2).

M0 is the initial place having token (black point) and M0 transit into M1 by usr1 transition. We can now verification some of the properties through reachability graph as follow:

- Reversibility: all places always come back to initial place.

- Reachability: all places are passable to transit from the initial place.

- Boundedness: marking count is always limited under 1.

- Liveness: the initial place always comes back without a deadlock.

Therefore we could identify the stability of the designed protocol.

From the reachability graph, all possible place sets are identified. Table IV summarizes the place set of the reachability graph. The header represents places and the 
left-most column represents the place set havin arking. The reachability analysis was very useful to validate state transitions of the places.

\section{CONCLUSION}

In this paper, we modeled the IEEE 802.16 MAC protocol using Higher-Order Stochastic Reward Net
(HOSRN). The correctness and consistency of the designed protocol is verified through Higher-Order Stochastic Reward Net modeling and the reachability graph, and the results of the reachability graph are checked by properties of HOSRN. This paper shows procedure of protocol verification, therefore, it is useful to apply the verification method using Higher-Order Petri-Net in other domains.

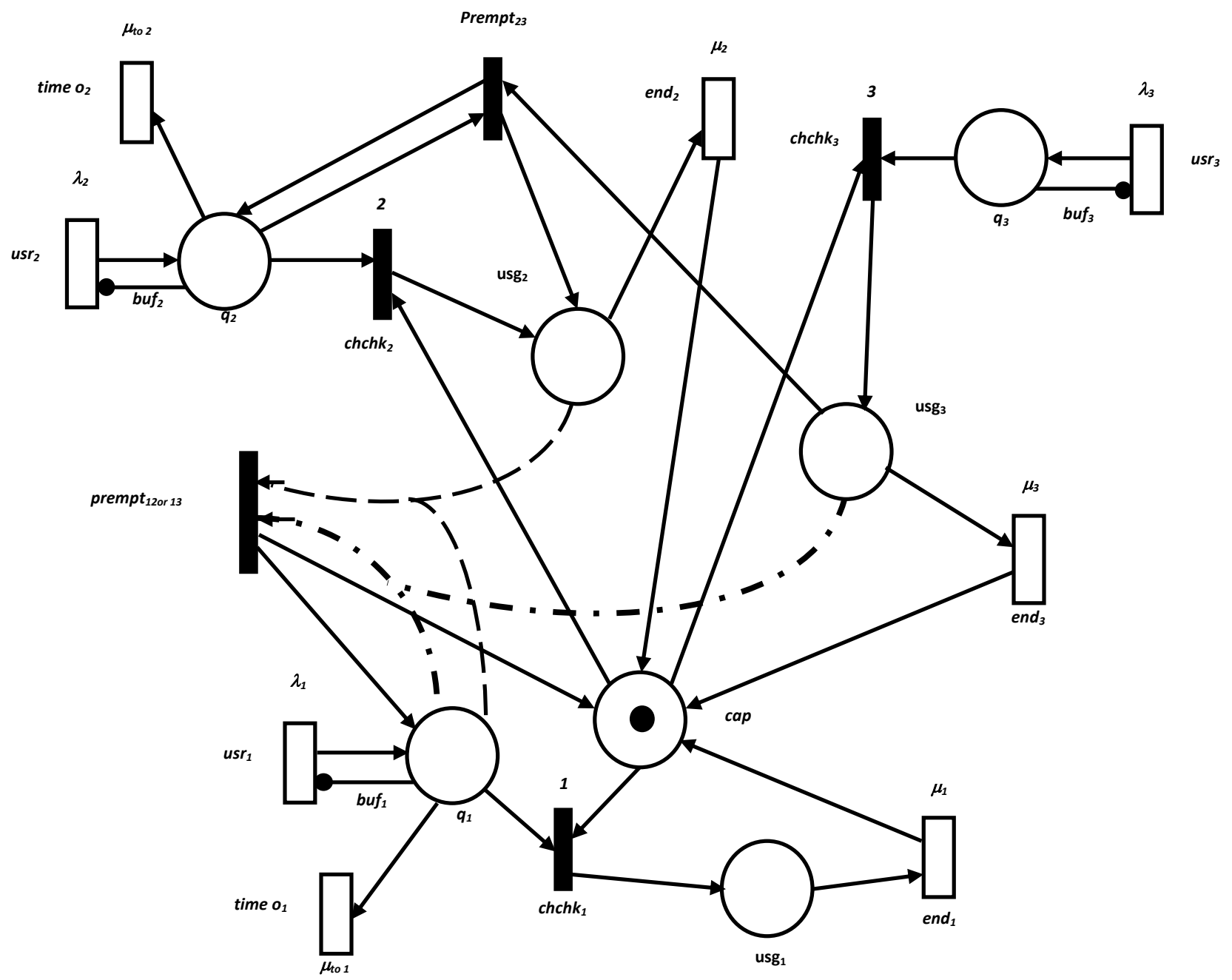

Figure 4: HOSRN model 


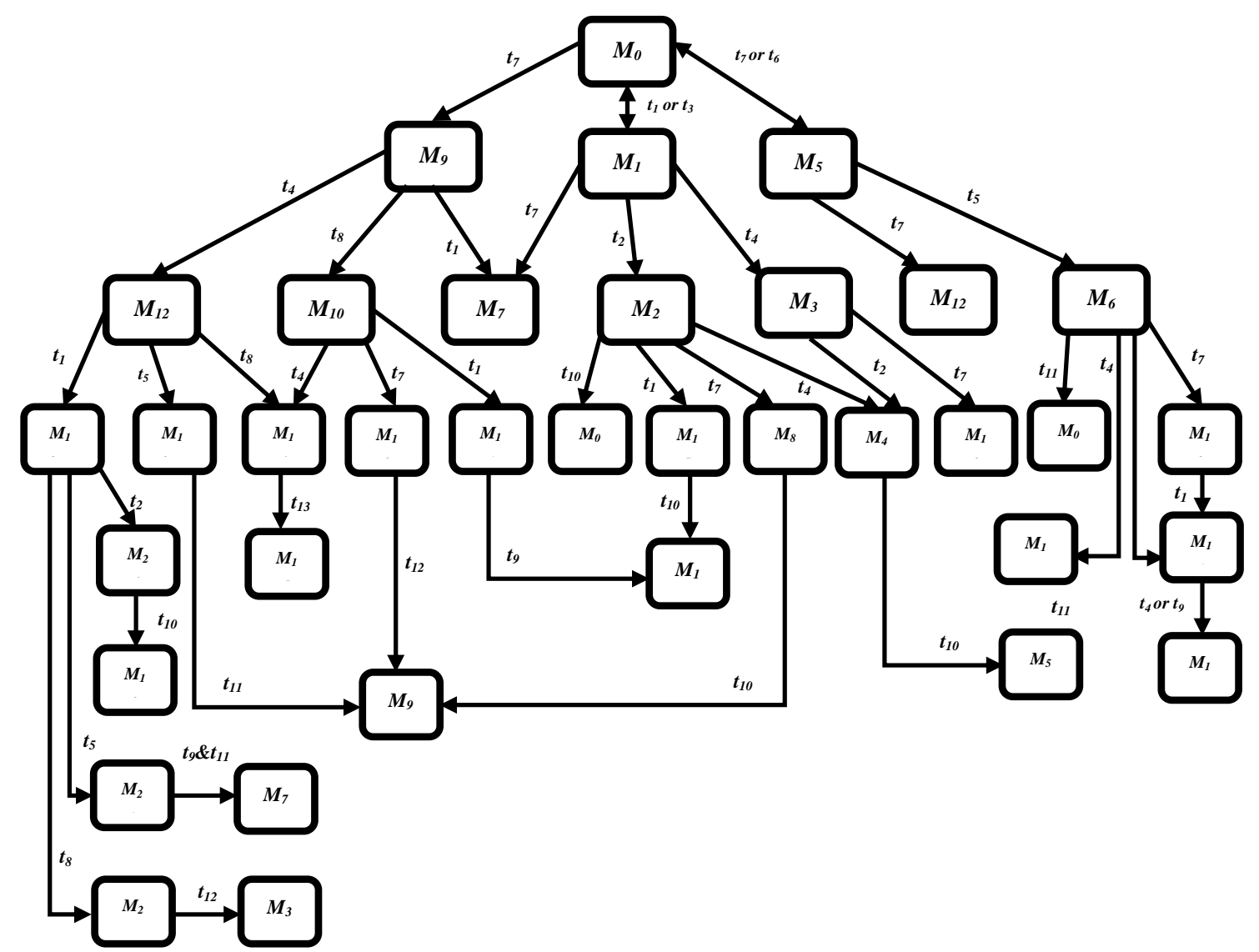

Figure 5: The reachability graph of the HOSRN model

TABLE IV: PLACE SET OF THE REACHABILITY GRAPH

\begin{tabular}{|c|c|c|c|c|c|c|c|}
\hline & $q_{1}$ & $U s g_{1}$ & $q_{2}$ & $\mathrm{usg}_{2}$ & $q_{3}$ & $\mathrm{usg}_{3}$ & cap \\
\hline $\boldsymbol{M}_{\boldsymbol{0}}$ & 0 & 0 & 0 & 0 & 0 & 0 & 1 \\
\hline$M_{1}$ & 1 & 0 & 0 & 0 & 0 & 0 & 1 \\
\hline$M_{2}$ & 0 & 1 & 0 & 0 & 0 & 0 & 0 \\
\hline$M_{3}$ & 1 & 0 & 1 & 0 & 0 & 0 & 1 \\
\hline$M_{4}$ & 0 & 1 & 1 & 0 & 0 & 0 & 0 \\
\hline$M_{5}$ & 0 & 0 & 1 & 0 & 0 & 0 & 1 \\
\hline$M_{6}$ & 0 & 0 & 0 & 1 & 0 & 0 & 0 \\
\hline $\boldsymbol{M}_{7}$ & 1 & 0 & 0 & 0 & 1 & 0 & 1 \\
\hline$M_{8}$ & 0 & 1 & 0 & 0 & 1 & 0 & 0 \\
\hline$M_{9}$ & 0 & 0 & 0 & 0 & 1 & 0 & 1 \\
\hline$M_{10}$ & 0 & 0 & 0 & 0 & 0 & 1 & 0 \\
\hline$M_{11}$ & 1 & 0 & 0 & 1 & 0 & 0 & 0 \\
\hline$M_{12}$ & 0 & 0 & 1 & 0 & 1 & 0 & 1 \\
\hline$M_{13}$ & 0 & 0 & 0 & 1 & 1 & 0 & 0 \\
\hline$M_{14}$ & 0 & 0 & 1 & 0 & 0 & 1 & 0 \\
\hline$M_{15}$ & 0 & 0 & 1 & 1 & 0 & 0 & 0 \\
\hline
\end{tabular}

\begin{tabular}{|l|l|l|l|l|l|l|l|}
\hline$M_{16}$ & 1 & 0 & 0 & 0 & 0 & 1 & 0 \\
\hline$M_{17}$ & 1 & 1 & 0 & 0 & 0 & 0 & 0 \\
\hline$M_{18}$ & 1 & 0 & 1 & 0 & 1 & 0 & 1 \\
\hline$M_{19}$ & 0 & 0 & 0 & 0 & 1 & 1 & 0 \\
\hline$M_{20}$ & 0 & 1 & 1 & 0 & 1 & 0 & 0 \\
\hline$M_{21}$ & 1 & 0 & 0 & 1 & 1 & 0 & 0 \\
\hline$M_{22}$ & 1 & 0 & 1 & 0 & 0 & 1 & 0 \\
\hline
\end{tabular}

\section{APPENDIX: DEFINITIONS OF PNS AND HOPNS}

Definition 1: A Petri Net (PN) is a bipartite directed graph with two types of nodes called places and transitions, that are connected by directed edges or directed arcs. Arcs exist only between places and transitions, i.e. there is no arc between two places or two transitions [7].

Each place may contain an arbitrary number of tokens. The number of tokens in a place is a non-negative integer. For a pictorial presentation, places are depicted as circles, transitions are represented by bars and tokens are represented by dots or integers in the places. Each directed arc in the bipartite graph is assigned a weight or a multiplicity, which is a natural number. If the multiplicity of an arc is not specified, then it is taken to be unity. 
Definition 2: For any transition in the PN, arcs directed out of the transition are called as output arcs of the transition. The corresponding places are called as output places of the transition.

Definition 3:For any transition in the PN, arcs directed into the transition are called input arcs of the transition. The corresponding places are called as input places of the transition.

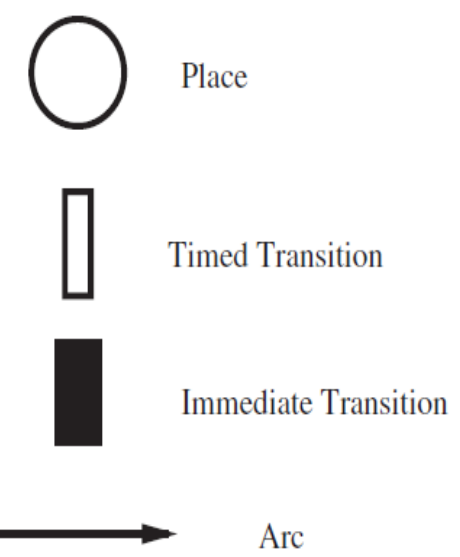

Figure 6: Components of an SRN.

Definition 4: A transition in a PN is said to be enabled if all of its input places have at least as many tokens as the multiplicities of the corresponding input arcs.

Definition 5: An inhibitor arc is an undirected arc (between a place and a transition) with multiplicity $\mathrm{k} \geq 1$ : If the multiplicity of the inhibitor arc is not specified, then it is taken to be unity. An inhibitor arc is pictorially represented as a shown in Figure 6.

If there exists an inhibitor arc with multiplicity $\mathrm{k}$ between a place and a transition, and if the place has $\mathrm{k}$ or more tokens, then the transition is inhibited even if it is enabled. When enabled (and not inhibited), a transition can fire. When a transition fires, it removes from each input place, the number of tokens given by multiplicities of the corresponding input arcs, and adds to each output place, the number of tokens given by the multiplicities of the corresponding output arcs.

Consider a PN containing $\mathrm{M}$ places and $\mathrm{N}$ transitions.

Definition 6:A marking, $\mathrm{M}(\mathrm{t})$ of a $\mathrm{PN}$ is an $\mathrm{M}$ tuple [ $\mathrm{m} 1(\mathrm{t}), \mathrm{m} 2(\mathrm{t}), \ldots, \mathrm{M}(\mathrm{t})]$ of non-negative integers, where $\operatorname{mi}(\mathrm{t})$ denotes the number of tokens in place $\mathrm{i}(1 \leq \mathrm{i} \leq \mathrm{M})$ at any given instant of time, $t$. For a given reference time instant, $\mathrm{t} 0 ; \mathrm{M}(\mathrm{t} 0)$ is called as the initial marking of the PN.

Definition 7: With respect to a given initial marking, the reachability set is the set of all markings that can be obtained from the initial marking through any possible firing sequences of transitions.

Definition 8: A Higher-Order Petri Net (HOPN) is formally defined in the same way as the classical Petri nets, $\mathrm{HOPN}=(\mathrm{P}, \mathrm{T}, \mathrm{F}, \mathrm{W}, \mathrm{M} 0)$ [4], where

- $F \subseteq\left(P_{1} \times T\right) \cap\left(P_{2} \times T\right) \cap \ldots \ldots \ldots \cap\left(P_{m} \times T\right) \cap(T \times P)$ is a set of arcs.
- $W: F \rightarrow N$ is a weight function;

- $M_{0}: P \rightarrow N$ is the initial token distribution, called the initial marking.

The main difference is the definition of the set of arcs and the weight.

Definition 9: A transition $t$ is said to be enabled or firable if there exist at least one of its kth-order input arcs such that each of this arc's places have at least as many tokens as the weight of this kth-order arc. Such an arc is defined as an enabled arc.

An enabled transition may or may not fire. When an enabled transition $t$ fires, one of its enabled arcs fires. The number of tokens in each of the input places related to the fired arc, $\mathrm{p}$, is reduced by the number that is equal to the weights assigned to the fired arc from $\mathrm{p}$ to $\mathrm{t}$. And the number of tokens in each of its output places increases by the number that is equal to the weights of the outgoing arc from the transition $t$.

\section{REFERENCES}

[1] H. M. Shamitha1, H. M. Guruprasad, Kishore. M and Ramesh. K, "Design and Implementation Of IEEE 802.16 Mac Layer Simulator", Int. J. Advances in Engineering \& Technology, Vol.5 (1), pp. 462-469, Nov.2012.

[2] S. Geetha and R. Jayaparvathy, " Modeling and Analysis of Bandwidth Allocation in IEEE 802.16 MAC: A Stochastic Reward Net Approach", Int. J. Communications, Network and System Sciences, Vol.3, pp. 631-637, July 2010.

[3] D. Lee and J. Baik, " QoS Protocol Verification using Petri-Net for Seamless Mobility in A Ubiquitous Environment: A Case Study", International Conference on Software Engineering, Artificial Intelligence, Networking, and Parallel/Distributed Computing, Phuket, pp. 617622 Aug.2008.

[4] T. Chow, J-Y Li, "Higher-Order Petri Net Models Based on Artificial Neural Networks", Artificial Intelligence, V. 92, pp. 289-300, 1997.

[5] W. Reisig and A. Meligy, "Using Modified Higher Order Petri Nets for Modeling Data Link Protocols", International Conference on Information Technology and Natural Sciences, 2003.

[6] M. Mahmoud, A. Meligy, A. Shafik and M. Hassan, "Design And Validity Analysis Of Asynchronous Processors Using Higher-Order Petri Nets", International Conference on Information Technology, 2005.

[7] R. Jayaparvathy, S. Anand, S. Dharmaraja and S. Sri-kanth, "Performance Analysis of IEEE 802.11 DCF with Stochastic Reward Nets," International Journal of Com-munication Systems, Vol. 20, No. 3, pp. 273-296, 2007.

[8] P.S.Revankar, A. S Kapse, and W. Z. Gandhare, "Overview of a MAC Scheduling Algorithm for IEEE 802.16 Wireless Networks", Int. J. Computer Applications, Vol.2, No.8, June 2010. 
[9] A. Khare, M. Saxena, R. S. Thakur and K. Chourasia, "Call Admission Control for Minimizing The Dropped Calls in CDMA Cellular Systems", Int. J. Latest Trends Computing, Vol.2, No.2, Sep., 2011.

Ali M. Meligy is a professor of computer science at the Menoufia University in Egypt. Previously, he was the head of computer science and information technology departments at Al-Hussein Bin Talal University in Jordan. his research interests include parallel processing and applications, distributed systems, Petri nets, and reuse-based software engineering.
Hani M. Ibrahim was born in Egypt on September 12th 1978. He received the M.S. and $\mathrm{PhD}$ degrees in Computer Science at the University of Menoufia, Egypt in 2004 and 2008, respectively. His research interests lies in the areas of image processing. Currently he is a lecture of Computer Science in the Faculty of Science, at the University of Menoufia, Egypt.

Amal M. Aqlan was born in Saudi Arabia March 7th 1978, He received the M.S. degrees in Computer Science at the University of Menoufia, Egypt in 2008. Ph.D. Currently she is Ph. D. candidate in Menoufia University, Department of Mathematics, Faculty of Science from Egypt.

How to cite this paper: Ali Mohammed Meligy, Hani Mohammed Ibrahim, Amal Mohammed Aqlan,"Modeling and Verification of 802.16 MAC Protocol using Higher-Order Petri Nets", IJCNIS, vol.6, no.4, pp.21-28, 2014. DOI: 10.5815/ijcnis.2014.04.03 